\title{
TOP LOCAL COHOMOLOGY MODULES OVER LOCAL RINGS AND THE WEAK GOING-UP PROPERTY
}

\author{
ASGHAR FAROKHI AND ALIREZA NAZARI
}

\begin{abstract}
Let $(R, \mathfrak{m})$ be a Noetherian local ring and let $\widehat{R}$ denote the $\mathfrak{m}$-adic completion of $R$. In this paper, we introduce the concept of the weak goingup property for the extension $R \subseteq \widehat{R}$ and we give some characterizations of this property. In particular, we show that this property is equivalent to the strong form of the Lichtenbaum-Hartshorne Vanishing Theorem. Also, when $R$ satisfies the weak going-up property, we show that for a finitely generated $R$-module $M$ of dimension $d$, and ideals $\mathfrak{a}$ and $\mathfrak{b}$ of $R$, we have $\operatorname{Att}_{R}\left(\mathrm{H}_{\mathfrak{a}}^{d}(M)\right)=$ $\operatorname{Att}_{R}\left(\mathrm{H}_{\mathfrak{b}}^{d}(M)\right)$ if and only if $\mathrm{H}_{\mathfrak{a}}^{d}(M) \cong \mathrm{H}_{\mathfrak{b}}^{d}(M)$, and we find a criterion for the cofiniteness of Artinian top local cohomology modules.
\end{abstract}

\section{INTRODUCTION}

Throughout this paper, let $(R, \mathfrak{m})$ be a Noetherian local ring and let $\widehat{R}$ denote the $\mathfrak{m}$-adic completion of $R$. For an $R$-module $M$, the $\mathfrak{m}$-adic completion of $M$ is denoted by $\widehat{M}$ and the $i$-th local cohomology module of $M$ with respect to $\mathfrak{a}$ is defined as

$$
\mathrm{H}_{\mathfrak{a}}^{i}(M)=\lim _{n \geq 1} \operatorname{Ext}_{R}^{i}\left(\frac{R}{\mathfrak{a}^{n}}, M\right) .
$$

For each Artinian $R$-module $A$, we denote by $\operatorname{Att}_{R} A$ the set of all attached prime ideals of $A$. Also, we denote $\{\mathfrak{p} \in \operatorname{Spec} R: \mathfrak{p} \supseteq \mathfrak{a}\}$ by $V(\mathfrak{a})$ and $\left\{\mathfrak{p} \in \operatorname{Ass}_{R}(M)\right.$ : $\left.\operatorname{dim} \frac{R}{\mathfrak{p}}=\operatorname{dim} M\right\}$ by $\operatorname{Assh}_{R}(M)$. For any ideal $\mathfrak{a}$ of $R$, the radical of $\mathfrak{a}$, denoted by $\sqrt{\mathfrak{a}}$, is defined to be the set $\left\{x \in R: x^{n} \in \mathfrak{a}\right.$ for some $\left.n \in \mathbb{N}\right\}$. Recall that an $R$-module $M$ is called $\mathfrak{a}$-cofinite if $\operatorname{Supp}(M) \subseteq V(\mathfrak{a})$ and $\operatorname{Ext}_{R}^{i}\left(\frac{R}{\mathfrak{a}}, M\right)$ is finitely generated for all $i \geq 0$. For any unexplained notation and terminology, we refer the reader to 3 ] and [10].

The Lichtenbaum-Hartshorne Vanishing Theorem [3, Theorem 8.2.1] gives necessary and sufficient conditions for the vanishing of certain top local cohomology modules by means of some conditions on the completion of $R$. Recently, some authors have tried to replace the conditions on $\widehat{R}$ by similar conditions on $R$. It is of course impossible in general. However, if $\widehat{R}$ is integral over $R$ or the extension

2010 Mathematics Subject Classification. 13D45; 14B15; 13E05.

Key words and phrases. Attached primes; Cofinite modules; Top local cohomology modules; Lichtenbaum-Hartshorne Vanishing Theorem. 
$R \subseteq \widehat{R}$ satisfies the going-up theorem, then we can do this (see [1, 4, 12]). In this article, we characterize local rings for which it is possible to check an analogous condition on the ring itself. For this work, we introduce the concept of the weak going-up property for $R$.

Definition 1.1. We say that $R$ satisfies the weak going-up property if for each $\beta \in \operatorname{Spec}(\widehat{R})$ with $\operatorname{dim} \frac{\widehat{R}}{\beta}=\operatorname{dim} \frac{R}{\beta \cap R}$, and each $\mathfrak{q} \in \operatorname{Spec}(R)$ with $\beta \cap R \subseteq \mathfrak{q}$ and $\operatorname{dim} \frac{R}{\mathfrak{q}}=1$, there exists $Q \in \operatorname{Spec}(\widehat{R})$ such that $\beta \subseteq Q$ and $Q \cap R=\mathfrak{q}$.

It is easy to see that, if the extension $R \subseteq \widehat{R}$ satisfies the going-up theorem (this holds, for instance, when $R \subseteq \widehat{R}$ is integral), then $R$ has the weak going-up property. By using a result of Charters and Loepp [6], we will show that the weak going-up property is different from the going-up property (see Example 2.2). In the main result of this paper, Theorem 2.4 we will show that the weak going-up property is equivalent to the strong form of the Lichtenbaum-Hartshorne Vanishing Theorem, which is defined as follows:

Definition 1.2. We say that the strong form of the Lichtenbaum-Hartshorne Vanishing Theorem holds over $R$, if for each finitely generated $R$-module $M$ of dimension $d$ and each proper ideal $\mathfrak{a}$ of $R$, the following statements are equivalent:

(i) $\mathrm{H}_{\mathfrak{a}}^{d}(M)=0$;

(ii) $\sqrt{\mathfrak{a}+\mathfrak{p}} \neq \mathfrak{m}$ for each prime ideal $\mathfrak{p} \in \operatorname{Assh}_{R}(M)$.

We provide two applications of Theorem 2.4 provided that $R$ satisfies the weak going-up property; on the one hand, we show (see Corollary 2.6 that, for a finitely generated $R$-module $M$ of dimension $d$ and ideals $\mathfrak{a}$ and $\mathfrak{b}$, we have that $\operatorname{Att}_{R}\left(\mathrm{H}_{\mathfrak{a}}^{d}(M)\right)=\operatorname{Att}_{R}\left(\mathrm{H}_{\mathfrak{b}}^{d}(M)\right)$ if and only if $\mathrm{H}_{\mathfrak{a}}^{d}(M) \cong \mathrm{H}_{\mathfrak{b}}^{d}(M)$. On the other hand, under the same assumptions on $M$, we give a criterion (see Corollary 2.8) for the $\mathfrak{b}$-cofiniteness of $\mathrm{H}_{\mathfrak{a}}^{d}(M)$. This result may be regarded as a stronger version of Melkersson's theorem of cofiniteness [11, Theorem 1.6] in the case of local cohomology modules over rings satisfying the weak going-up property.

\section{MAin Results}

In this section, we first present an example of a local ring which shows that the weak going-up property is different from the going-up property. Recall that the extension $R \subseteq \widehat{R}$ satisfies the going-up theorem if given two prime ideals $\mathfrak{p} \subset \mathfrak{q}$ of $R$ and a prime ideal $\beta$ of $\widehat{R}$ lying over $\mathfrak{p}$, there exists a prime ideal $Q$ of $\widehat{R}$ such that $\beta \subset Q$ and $Q \cap R=\mathfrak{q}$. In order to give an example of such a local ring, we first recall a result of Charters and Loepp [6, Theorem 3.1 and Lemma 2.8].

Proposition 2.1 (Charters-Loepp). Let $\left(T, \mathfrak{m}_{T}\right)$ be a complete local ring. Let $W \subset \operatorname{Spec}(T)$ be a non-empty subset of prime ideals which satisfies:

(i) $W$ has finitely many maximal elements;

(ii) $\mathfrak{m}_{T} \notin W$ and $\operatorname{Ass}(T) \subseteq W$;

(iii) if $P \in \operatorname{Spec}(T), Q \in W$, and $P \subseteq Q$ then $P \in W$;

(iv) for any $P \in W, P \cap \mathbb{Z} .1_{T}=0$. 
Then there is a Noetherian local domain $(R, \mathfrak{m})$ such that $T$ is isomorphic to the $\mathfrak{m}$-adic completion of $R$ and the generic formal fiber of $R, \tau^{-1}(0)=W$. Moreover, if $\mathfrak{p}$ is a prime ideal of $R, \mathfrak{p} \neq 0$, then $T \otimes_{R} k(\mathfrak{p}) \cong k(\mathfrak{p})$, where $k(\mathfrak{p})=\frac{R_{\mathfrak{p}}}{\mathfrak{p} R_{\mathfrak{p}}}$.

Using this result, we are able to give the following example.

Example 2.2. Let $\mathbb{Q}$ be a field of rational numbers. According to the above Proposition, set $T:=\mathbb{Q} \llbracket x, y \rrbracket, \mathfrak{m}_{T}:=(x, y)$, and $W:=\{0,(x)\}$. Then there exists a Noetherian local domain $(R, \mathfrak{m})$ such that $T \cong \widehat{R}$. It is easy to see that in this case $R$ has the weak going-up property. Consider the prime ideal $(x)$ of $\widehat{R}$; we have $(x) \cap R=0$. Now let $\mathfrak{q}$ be a prime ideal of $R$ such that $\operatorname{dim} \frac{R}{\mathfrak{q}}=1$; then it is clear that there is no prime ideal $Q$ containing $(x)$ and lying over $\mathfrak{q}$. It follows that $R$ has no going-up property.

Before proving Theorem 2.4 we need the following lemma which is proved in [5].

Lemma 2.3 (See [5, Lemma 4.3]). Let $M$ be a finitely generated $R$-module and let $\mathfrak{q}$ be a prime ideal of $R$ such that $\mathfrak{q} \supseteq \operatorname{Ann}_{R}\left(\mathrm{H}_{\mathfrak{m}}^{\operatorname{dim} M}(M)\right)$ and $\operatorname{dim} M_{\mathfrak{q}}=\operatorname{dim} M-$ $\operatorname{dim} \frac{R}{\mathfrak{q}}$. Then $\operatorname{Ann}_{R}\left(0:_{\mathrm{H}_{\mathfrak{m}}^{\operatorname{dim}} M(M)} \mathfrak{q}\right)=\mathfrak{q}$.

The next theorem is the main result of this paper.

Theorem 2.4. The following statements are equivalent:

(i) $\operatorname{Att}_{R}\left(\mathrm{H}_{\mathfrak{a}}^{\operatorname{dim} M}(M)\right)=\{\mathfrak{p} \in \operatorname{Assh}(M): \sqrt{\mathfrak{p}+\mathfrak{a}}=\mathfrak{m}\}$ for each finitely generated $R$-module $M$ and each proper ideal $\mathfrak{a}$ of $R$;

(ii) the strong form of the Lichtenbaum-Hartshorne Vanishing Theorem holds over $R$;

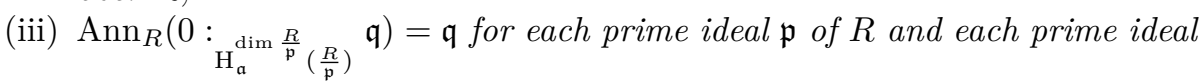
$\mathfrak{q} \supseteq \operatorname{Ann}_{R}\left(\mathrm{H}_{\mathfrak{a}}^{\operatorname{dim} \frac{R}{\mathfrak{p}}}\left(\frac{R}{\mathfrak{p}}\right)\right)$ with $\operatorname{dim} \frac{R}{\mathfrak{q}}=1 ;$

(iv) $R$ satisfies the weak going-up property.

Proof. (i) $\Rightarrow$ (ii) It is clear.

(ii) $\Rightarrow$ (iii) For $\mathfrak{p} \in \operatorname{Spec}(R)$, let $\mathfrak{q}$ be a prime ideal of $R$ such that $\operatorname{dim} \frac{R}{\mathfrak{q}}=1$ and $\mathfrak{q} \supseteq \operatorname{Ann}_{R}\left(\mathrm{H}_{\mathfrak{a}}^{\operatorname{dim} \frac{R}{\mathfrak{p}}}\left(\frac{R}{\mathfrak{p}}\right)\right)$. Since $\mathrm{H}_{\mathfrak{a}}^{\operatorname{dim} \frac{R}{\mathfrak{p}}}\left(\frac{R}{\mathfrak{p}}\right) \neq 0$, it follows from statement (ii) that $\sqrt{\mathfrak{p}+\mathfrak{a}}=\mathfrak{m}$ and so $\mathrm{H}_{\mathfrak{a}}^{\operatorname{dim} \frac{R}{\mathfrak{p}}}\left(\frac{R}{\mathfrak{p}}\right) \cong \mathrm{H}_{\mathfrak{m}}^{\operatorname{dim} \frac{R}{\mathfrak{p}}}\left(\frac{R}{\mathfrak{p}}\right)$. Therefore the proof is complete if we

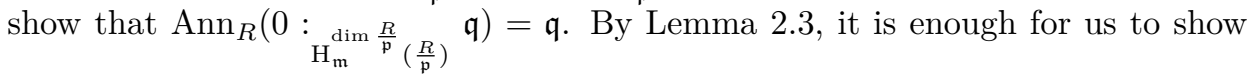
that

$$
\operatorname{dim}\left(\frac{R}{\mathfrak{p}}\right)_{\mathfrak{q}}=\operatorname{dim}\left(\frac{R}{\mathfrak{p}}\right)-\operatorname{dim} \frac{R}{\mathfrak{q}}=\operatorname{dim} \frac{R}{\mathfrak{p}}-1 .
$$

Suppose, on the contrary, that $\operatorname{dim}\left(\frac{R}{\mathfrak{p}}\right)_{\mathfrak{q}}<\operatorname{dim}\left(\frac{R}{\mathfrak{p}}\right)-1$, and look for a contradiction. By the Flat Base Change Theorem [3, Theorem 4.3.2] and Grothendieck's Vanishing Theorem [3, Theorem 6.1.2], we have

$$
\left(\mathrm{H}_{\mathfrak{q}}^{\operatorname{dim} \frac{R}{\mathfrak{p}}-1}\left(\frac{R}{\mathfrak{p}}\right)\right)_{\mathfrak{q}} \cong \mathrm{H}_{\mathfrak{q} R_{\mathfrak{q}}}^{\operatorname{dim} \frac{R}{\mathfrak{p}}-1}\left(\left(\frac{R}{\mathfrak{p}}\right)_{\mathfrak{q}}\right)=0 .
$$


Since $\operatorname{Supp}\left(\mathrm{H}_{\mathfrak{q}}^{\operatorname{dim} \frac{R}{\mathfrak{p}}-1}\left(\frac{R}{\mathfrak{p}}\right)\right) \subseteq V(\mathfrak{q})$, it follows that $\operatorname{Supp}\left(\mathrm{H}_{\mathfrak{q}}^{\operatorname{dim} \frac{R}{\mathfrak{p}}-1}\left(\frac{R}{\mathfrak{p}}\right)\right) \subseteq\{\mathfrak{m}\}$ and so for $x \in \mathfrak{m}-\mathfrak{q}$ we have $\left(\mathrm{H}_{\mathfrak{q}}^{\operatorname{dim} \frac{R}{\mathfrak{p}}-1}\left(\frac{R}{\mathfrak{p}}\right)\right)_{x}=0$. Since $\mathfrak{q} \supseteq \operatorname{Ann}_{R}\left(\mathrm{H}_{\mathfrak{a}}^{\operatorname{dim} \frac{R}{\mathfrak{p}}}\left(\frac{R}{\mathfrak{p}}\right)\right) \supseteq \mathfrak{p}$, by statement (ii) we have $\mathrm{H}_{\mathfrak{q}}^{\operatorname{dim} \frac{R}{\mathfrak{p}}}\left(\frac{R}{\mathfrak{p}}\right)=0$. It now follows from the long exact sequence [3. Proposition 8.1.2]

$$
\left(\mathrm{H}_{\mathfrak{q}}^{\operatorname{dim} \frac{R}{\mathfrak{p}}-1}\left(\frac{R}{\mathfrak{p}}\right)\right)_{x} \longrightarrow \mathrm{H}_{\mathfrak{q}+R x}^{\operatorname{dim} \frac{R}{\mathfrak{p}}}\left(\frac{R}{\mathfrak{p}}\right) \longrightarrow \mathrm{H}_{\mathfrak{q}}^{\operatorname{dim} \frac{R}{\mathfrak{p}}}\left(\frac{R}{\mathfrak{p}}\right) \longrightarrow 0
$$

that $\mathrm{H}_{\mathfrak{m}}^{\operatorname{dim} \frac{R}{\mathfrak{p}}}\left(\frac{R}{\mathfrak{p}}\right) \cong \mathrm{H}_{\mathfrak{q}+R x}^{\operatorname{dim} \frac{R}{\mathfrak{p}}}\left(\frac{R}{\mathfrak{p}}\right)=0$, which is a contradiction to the Non-Vanishing Theorem [3, Theorem 6.1.4].

(iii) $\Rightarrow$ (iv) Let $\beta \in \operatorname{Spec}(\widehat{R})$ with $\operatorname{dim} \frac{\widehat{R}}{\beta}=\operatorname{dim} \frac{R}{\beta \cap R}$, and let $\mathfrak{q} \in \operatorname{Spec}(R)$ with $\beta \cap R \subseteq \mathfrak{q}$ and $\operatorname{dim} \frac{R}{\mathfrak{q}}=1$. We must show that there exists $Q \in \operatorname{Spec}(\widehat{R}) \operatorname{such}$ that $Q \supseteq \beta$ and $Q \cap R=\mathfrak{q}$. Suppose, on the contrary, that there does not exist such a prime ideal and look for a contradiction. It follows that $\sqrt{\mathfrak{q} \widehat{R}+\beta}=\widehat{\mathfrak{m}}$ and so by the Lichtenbaum-Hartshorne Vanishing Theorem [3, Theorem 8.2.1] we

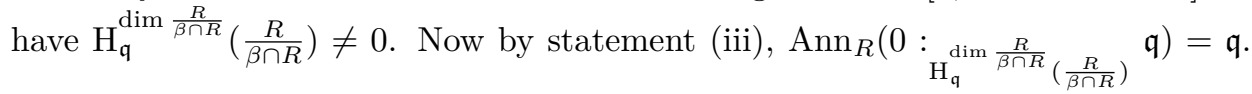
But, by [7, Theorem 3], the Artinian $R$-module $\mathrm{H}_{\mathfrak{q}}^{\operatorname{dim} \frac{R}{\beta \cap R}}\left(\frac{R}{\beta \cap R}\right)$ is $\mathfrak{q}$-cofinite, and

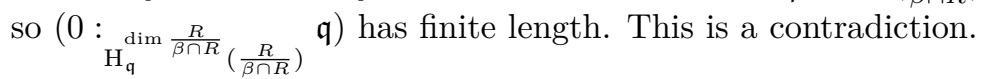

(iv) $\Rightarrow$ (i) By [3, 8.2.4 and 8.2.5], we have

$$
\operatorname{Att}_{R}\left(\mathrm{H}_{\mathfrak{a}}^{d}(M)\right)=\left\{\beta \cap R \mid \beta \in \operatorname{Att}_{\widehat{R}}\left(\mathrm{H}_{\mathfrak{a}}^{d}(M)\right)\right\}
$$

[8, Corollary 3.3] $=\left\{\beta \cap R \mid \beta \in \operatorname{Assh}_{\widehat{R}}(\widehat{M}), \sqrt{\beta+\mathfrak{a} \widehat{R}}=\widehat{\mathfrak{m}}\right\}$

$\left[\right.$ by statement (iv)] $=\left\{\mathfrak{p} \in \operatorname{Assh}_{R}(M) \mid \sqrt{\mathfrak{p}+\mathfrak{a}}=\mathfrak{m}\right\}$.

In the next corollary, we give some conditions for which the weak going-up property is equivalent to the going-up property. For each $\mathfrak{p} \in \operatorname{Spec}(R)$, denote by $\alpha(R, \mathfrak{p})$ the Krull dimension of the fiber ring $\widehat{R} \otimes_{R} k(\mathfrak{p})$, where $k(\mathfrak{p}):=\frac{R_{\mathfrak{p}}}{\mathfrak{p} R_{\mathfrak{p}}}$, and $\alpha(R):=\max \{\alpha(R, \mathfrak{p}): \mathfrak{p} \in \operatorname{Spec}(R)\}$.

Corollary 2.5. Let $(R, \mathfrak{m})$ be a universally catenary Noetherian local ring with $\alpha(R)=0$. Then the following statements are equivalent:

(i) $R$ has the weak going-up property;

(ii) the extension $R \subseteq \widehat{R}$ satisfies the going-up theorem.

Proof. Under these assumptions, Cuong proved [4, Theorem 3.2] that the strong form of the Lichtenbaum-Hartshorne Vanishing Theorem holds over $R$ if and only if $R \subseteq \widehat{R}$ satisfies the going-up property. Keeping in mind this fact, the conclusion follows immediately from Theorem 2.4

As an application of Theorem 2.4 we show that if $R$ satisfies the weak going-up property, then for a finitely generated $R$-module $M$ of dimension $d$, and ideals $\mathfrak{a}$ 
and $\mathfrak{b}$ of $R$, we have $\operatorname{Att}_{R}\left(\mathrm{H}_{\mathfrak{a}}^{d}(M)\right)=\operatorname{Att}_{R}\left(\mathrm{H}_{\mathfrak{b}}^{d}(M)\right)$ if and only if $\mathrm{H}_{\mathfrak{a}}^{d}(M) \cong \mathrm{H}_{\mathfrak{b}}^{d}(M)$. This is a generalization of [9, Proposition 1.5].

Corollary 2.6. If $R$ satisfies the weak going-up property, then for a finitely generated $R$-module $M$ of dimension $d$, and ideals $\mathfrak{a}$ and $\mathfrak{b}$ of $R$, the following statements are equivalent:

(i) $\operatorname{Att}_{R}\left(\mathrm{H}_{\mathfrak{a}}^{d}(M)\right)=\operatorname{Att}_{R}\left(\mathrm{H}_{\mathfrak{b}}^{d}(M)\right)$;

(ii) $\operatorname{Att}_{\widehat{R}}\left(\mathrm{H}_{\mathfrak{a}}^{d}(M)\right)=\operatorname{Att}_{\widehat{R}}\left(\mathrm{H}_{\mathfrak{b}}^{d}(M)\right)$;

(iii) $\mathrm{H}_{\mathfrak{a}}^{d}(M) \cong \mathrm{H}_{\mathfrak{b}}^{d}(M)$.

Proof. (i) $\Rightarrow\left(\right.$ ii) Let $\beta \in \operatorname{Att}_{\widehat{R}}\left(\mathrm{H}_{\mathfrak{a}}^{d}(M)\right)$, then by [8, Corollary 3.3] and [3, 8.2.4 and 8.2.5], we have $\beta \in \operatorname{Assh}_{\widehat{R}}(\widehat{M})$ and $\beta \cap R \in \operatorname{Att}_{R}\left(\mathrm{H}_{\mathfrak{a}}^{d}(M)\right)$. Hence, by assumption, $\beta \cap R \in \operatorname{Att}_{R}\left(\mathrm{H}_{\mathfrak{b}}^{d}(M)\right)$. It follows from Theorem 2.4 that $\sqrt{\beta \cap R+\mathfrak{b}}=\mathfrak{m}$ and so

$$
\mathfrak{m} \widehat{R}=(\sqrt{\beta \cap R+\mathfrak{b}}) \widehat{R} \subseteq \sqrt{(\beta \cap R+\mathfrak{b}) \widehat{R}}=\sqrt{(\beta \cap R) \widehat{R}+\mathfrak{b} \widehat{R}} \subseteq \sqrt{\beta+\mathfrak{b} \widehat{R}} \subseteq \widehat{\mathfrak{m}}
$$

Now, it follows from [8, Corollary 3.3] that $\beta \in \operatorname{Att}_{\widehat{R}}\left(\mathrm{H}_{\mathfrak{b}}^{d}(M)\right)$. A similar argument shows that $\operatorname{Att}_{\widehat{R}}\left(\mathrm{H}_{\mathfrak{b}}^{d}(M)\right) \subseteq \operatorname{Att}_{\widehat{R}}\left(\mathrm{H}_{\mathfrak{a}}^{d}(M)\right)$.

(ii) $\Rightarrow$ (iii) Set $X=\operatorname{Att}_{\widehat{R}}\left(\mathrm{H}_{\mathfrak{a}}^{d}(M)\right)=\operatorname{Att}_{\widehat{R}}\left(\mathrm{H}_{\mathfrak{b}}^{d}(M)\right)$. Then by [8, Corollary 3.3] we have $X=\left\{\beta \in \operatorname{Assh}_{\widehat{R}}(\widehat{M}): \sqrt{\beta+\mathfrak{a} \widehat{R}}=\widehat{\mathfrak{m}}\right\}=\left\{\beta \in \operatorname{Assh}_{\widehat{R}}(\widehat{M}): \sqrt{\beta+\mathfrak{b} \widehat{R}}=\widehat{\mathfrak{m}}\right\}$. Note that we have $\mathrm{H}_{\mathfrak{a}}^{d}(M) \cong \mathrm{H}_{\mathfrak{a}}^{d}(M) \otimes_{R} \widehat{R} \cong \mathrm{H}_{\mathfrak{a} \widehat{R}}^{d}(\widehat{M})$. There exists an $\widehat{R}$-submodule $N$ of $\widehat{M}$ such that $\operatorname{Ass}_{\widehat{R}}(N)=\operatorname{Ass}_{\widehat{R}}(\widehat{M}) \backslash X$ and $\operatorname{Ass}_{\widehat{R}}(\widehat{M} / N)=X$ (see [2, p. 263, Proposition 4]). By the choice of $N$, it is easy to check that $\sqrt{\operatorname{Ann}_{\widehat{R}}(\widehat{M} / N)+\mathfrak{a} \widehat{R}}=$ $\widehat{\mathfrak{m}}$. Now by [8, Corollary 3.3] we have $\mathrm{H}_{\mathfrak{a} \widehat{R}}^{d}(N)=0$. It follows that

$$
\mathrm{H}_{\mathfrak{a}}^{d}(M) \cong \mathrm{H}_{\mathfrak{a} \widehat{R}}^{d}(\widehat{M}) \cong \mathrm{H}_{\mathfrak{a} \widehat{R}}^{d}(\widehat{M} / N) \cong \mathrm{H}_{\mathfrak{a} \widehat{R}+\operatorname{Ann}_{\widehat{R}}(\widehat{M} / N)}(\widehat{M} / N) \cong \mathrm{H}_{\mathfrak{m} \widehat{R}}^{d}(\widehat{M} / N) .
$$

A similar argument shows that

$$
\begin{aligned}
& \mathrm{H}_{\mathfrak{b}}^{d}(M) \cong \mathrm{H}_{\mathfrak{b} \widehat{R}}^{d}(\widehat{M}) \cong \mathrm{H}_{\mathfrak{b} \widehat{R}}^{d}(\widehat{M} / N) \cong \mathrm{H}_{\mathfrak{b} \widehat{R}+\operatorname{Ann}_{\widehat{R}}(\widehat{M} / N)}^{d}(\widehat{M} / N) \cong \mathrm{H}_{\mathfrak{m} \widehat{R}}^{d}(\widehat{M} / N) . \\
& \text { (iii) } \Rightarrow \text { (i) is clear. }
\end{aligned}
$$

The next example shows that if $R$ does not have the weak going-up property, then the result of Corollary 2.6 may not be true even when $R$ is a quotient of a regular local ring.

Example 2.7. Let $K$ be a field of characteristic 0 . Let $R^{\prime}:=K\left[X_{1}, X_{2}, X_{3}\right]$, $\mathfrak{m}^{\prime}:=\left(X_{1}, X_{2}, X_{3}\right)$, and $\mathfrak{b}=\left(X_{2}^{2}-X_{1}^{2}-X_{1}^{3}\right)$. Set $R:=\left(\frac{R^{\prime}}{\mathfrak{b}}\right)_{\frac{\mathfrak{m}^{\prime}}{\mathfrak{b}}}$ and let $\mathfrak{q}$ be the extension of the ideal

$$
\left(X_{1}+X_{2}-X_{2} X_{3},\left(X_{3}-1\right)^{2}\left(X_{1}+1\right)-1\right)
$$

of $R^{\prime}$ to $R$. Then $R$ is a 2-dimensional local domain, and $\mathfrak{q}$ is a prime ideal of $R$ with $\operatorname{dim} \frac{R}{q}=1$ (see [3, Exercise 8.2.9]). Now by [7, Theorem 3] the Artinian $R$-module $\mathrm{H}_{\mathfrak{q}}^{2}(R)$ is $\mathfrak{q}$-cofinite, and so $\left(0:_{\mathrm{H}_{\mathfrak{q}}^{2}(R)} \mathfrak{q}\right)$ has finite length. It follows that 
$\operatorname{Ann}_{R}\left(0:_{\mathrm{H}_{\mathfrak{q}}^{2}(R)} \mathfrak{q}\right) \neq \mathfrak{q}$ and so, by Theorem 2.4 $R$ does not have the weak goingup property. Now let $\mathfrak{m}$ denote the maximal ideal of the local ring $R$, then by Lemma 2.3 we have $\operatorname{Ann}_{R}\left(0:_{\mathrm{H}_{\mathfrak{m}}^{2}(R)} \mathfrak{q}\right)=\mathfrak{q}$. It follows that $\mathrm{H}_{\mathfrak{q}}^{2}(R)$ and $\mathrm{H}_{\mathfrak{m}}^{2}(R)$ are not isomorphic. However, $\operatorname{Att}_{R}\left(\mathrm{H}_{\mathfrak{q}}^{2}(R)\right)=\operatorname{Att}_{R}\left(\mathrm{H}_{\mathfrak{m}}^{2}(R)\right)=\{0\}$.

As another application of Theorem 2.4. we prove the following result.

Corollary 2.8. If $R$ satisfies the weak going-up property, then for a finitely generated $R$-module $M$ of dimension $d$, and ideals $\mathfrak{a}$ and $\mathfrak{b}$ of $R$, the following statements are equivalent:

(i) $\mathrm{H}_{\mathfrak{a}}^{d}(M)$ is $\mathfrak{b}$-cofinite;

(ii) $\operatorname{Hom}_{R}\left(\frac{R}{\mathfrak{b}}, \mathrm{H}_{\mathfrak{a}}^{d}(M)\right)$ has finite length;

(iii) $\operatorname{Att}_{R}\left(\mathrm{H}_{\mathfrak{a}}^{d}(M)\right) \subseteq \operatorname{Att}_{R}\left(\mathrm{H}_{\mathfrak{b}}^{d}(M)\right)$;

(iv) $\sqrt{\mathfrak{b}+\mathfrak{p}}=\mathfrak{m}$, for each $\mathfrak{p} \in \operatorname{Att}_{R}\left(\mathrm{H}_{\mathfrak{a}}^{d}(M)\right)$;

(v) $\sqrt{\mathfrak{b}+\operatorname{Ann}_{R}\left(\mathrm{H}_{\mathfrak{a}}^{d}(M)\right)}=\mathfrak{m}$.

Proof. (i) $\Rightarrow$ (ii) It is trivial.

(ii) $\Rightarrow$ (iii) Suppose on the contrary that $\operatorname{Att}_{R}\left(\mathrm{H}_{\mathfrak{a}}^{d}(M)\right) \nsubseteq \operatorname{Att}_{R}\left(\mathrm{H}_{\mathfrak{b}}^{d}(M)\right)$. So, by Theorem 2.4. there exists $\mathfrak{p} \in \operatorname{Att}_{R}\left(\mathrm{H}_{\mathfrak{a}}^{d}(M)\right)$ such that $\sqrt{\mathfrak{b}+\mathfrak{p}} \neq \mathfrak{m}$. Now, let $\mathfrak{q}$ be a prime ideal of $R$ such that $\sqrt{\mathfrak{b}+\mathfrak{p}} \subseteq \mathfrak{q}$ and $\operatorname{dim} \frac{R}{\mathfrak{q}}=1$. By assumption (ii), $\operatorname{Hom}_{R}\left(\frac{R}{\mathfrak{q}}, \mathrm{H}_{\mathfrak{a}}^{d}(M)\right)$ has finite length. It follows that $\operatorname{Hom}_{\widehat{R}}\left(\frac{\widehat{R} \widehat{R}}{\widehat{R}}, \mathrm{H}_{\mathfrak{a} \widehat{R}}^{d}(\widehat{M})\right)$ has finite length as an $\widehat{R}$-module. By [3, 11.3.7], there exists a prime ideal $\beta \in \operatorname{Att}_{\widehat{R}}\left(\mathrm{H}_{\mathfrak{a} \widehat{R}}^{d}(\widehat{M})\right)$ such that $\beta \cap R=\mathfrak{p}$. Note that $\operatorname{dim} \frac{\widehat{R}}{\beta}=\operatorname{dim} \frac{R}{\mathfrak{p}}=d$. Since $R$ has the weak going-up property, there exists a prime ideal $Q$ of $\widehat{R}$ such that $Q \cap R=\mathfrak{q}$ and $\beta \subseteq Q$. It is clear that $\operatorname{Ann}_{\widehat{R}}\left(\mathrm{H}_{\mathfrak{a} \widehat{R}}^{d}(\widehat{M})\right) \subseteq Q$. So, it follows by Matlis duality and [3, 10.2.16] that

$$
\begin{aligned}
\operatorname{Ann}_{\widehat{R}}\left(\operatorname{Hom}_{\widehat{R}}\left(\frac{\widehat{R}}{Q}, \mathrm{H}_{\mathfrak{a} \widehat{R}}^{d}(\widehat{M})\right)\right) & =\operatorname{Ann}_{\widehat{R}}\left(\operatorname{Hom}_{\widehat{R}}\left(\operatorname{Hom}_{\widehat{R}}\left(\frac{\widehat{R}}{Q}, \mathrm{H}_{\mathfrak{a} \widehat{R}}^{d}(\widehat{M})\right), E\left(\frac{\widehat{R}}{\widehat{\mathfrak{m}}}\right)\right)\right) \\
& =\operatorname{Ann}_{\widehat{R}}\left(\frac{\widehat{R}}{Q} \otimes_{\widehat{R}} \operatorname{Hom}_{\widehat{R}}\left(\mathrm{H}_{\mathfrak{a} \widehat{R}}^{d}(\widehat{M}), E\left(\frac{\widehat{R}}{\widehat{\mathfrak{m}}}\right)\right)\right)=Q .
\end{aligned}
$$

This shows that $\operatorname{Hom}_{\widehat{R}}\left(\frac{\widehat{R}}{\mathfrak{q} R}, \mathrm{H}_{\mathfrak{a} \widehat{R}}^{d}(\widehat{M})\right)$ does not have finite length, which is a contradiction.

(iii) $\Rightarrow$ (iv) It follows from Theorem 2.4 .

(iv) $\Rightarrow(\mathrm{v})$ and $(\mathrm{v}) \Rightarrow(\mathrm{i})$ are clear.

Remark 2.9. If $R$ does not have the weak going-up property, then by Theorem 2.4 there exist a finitely generated $R$-module $M$ of dimension $d$ and ideal $\mathfrak{a}$ of $R$ such that $\operatorname{Att}_{R}\left(\mathrm{H}_{\mathfrak{a}}^{\operatorname{dim} M}(M)\right) \neq\{\mathfrak{p} \in \operatorname{Assh}(M): \sqrt{\mathfrak{p}+\mathfrak{a}}=\mathfrak{m}\}$. It follows that there exists $\mathfrak{p} \in \operatorname{Att}_{R}\left(\mathrm{H}_{\mathfrak{a}}^{\operatorname{dim} M}(M)\right)$ such that $\sqrt{\mathfrak{p}+\mathfrak{a}} \neq \mathfrak{m}$. In Corollary 2.8 set $\mathfrak{a}=\mathfrak{b}$. Note that, by [7, Theorem 3], $\mathrm{H}_{\mathfrak{a}}^{\operatorname{dim} M}(M)$ is $\mathfrak{a}$-cofinite but there exists $\mathfrak{p} \in \operatorname{Att}_{R}\left(\mathrm{H}_{\mathfrak{a}}^{\operatorname{dim} M}(M)\right)$ such that $\sqrt{\mathfrak{p}+\mathfrak{a}} \neq \mathfrak{m}$. This shows that the condition that $R$ has the weak going-up property is necessary in Corollary 2.8 (and so it can not be omitted). 


\section{ACKNOWLEDGEMENT}

The authors are deeply grateful to the referee for his/her careful reading of the paper and valuable suggestions.

\section{REFERENCES}

[1] N. Abazari and K. Bahmanpour, A note on the artinian cofinite modules, Comm. Algebra 42 (2014), no. 3, 1270-1275. MR 3169628.

[2] N. Bourbaki, Elements of Mathematics. Commutative Algebra, Addison-Wesley, Reading, Mass., 1972. MR 0360549

[3] M. P. Brodmann and R. Y. Sharp, Local Cohomology: An Algebraic Introduction with Geometric Applications. Cambridge University Press, Cambridge, 1998. MR 1613627

[4] D. T. Cuong, Local rings with zero-dimensional formal fibers, J. Algebra 403 (2014), 77-92. MR 3166066

[5] N. T. Cuong, N. T. Dung and L. T. Nhan, Top local cohomology and the catenaricity of the unmixed support of a finitely generated module, Comm. Algebra 35 (2007), no. 5, 1691-1701. MR 2317638

[6] P. Charters and S. Loepp, Semilocal generic formal fibers, J. Algebra 278 (2004), no. 1, 370-382. MR 2068083

[7] D. Delfino and T. Marley, Cofinite modules and local cohomology, J. Pure and Appl. Algebra 121 (1997), no. 1, 45-52. MR 1471123

[8] K. Divaani-Aazar and P. Schenzel, Ideal topologies, local cohomology and connectedness, Math. Proc. Cambridge Philos. Soc. 131 (2001), no. 2, 211-226. MR 1857116

[9] M. T. Dibaei and S. Yassemi, Top local cohomology modules, Algebra Colloq. 14 (2007), no. 2, 209-214. MR 2305389

[10] H. Matsumura, Commutative Ring Theory, Cambridge University Press, Cambridge, 1986. MR 0879273

[11] L. Melkersson, Properties of cofinite modules and applications to local cohomology. Math. Proc. Cambridge Philos. Soc. 125 (1999), no. 3, 417-423. MR 1656785

[12] H. Zöschinger, Über die Bedingung Going up für $R \subset \widehat{R}$, Arch. Math. (Basel) 95 (2010), no. 3, 225-231. MR 2719380

\section{A. Farokhi}

Faculty of Mathematical Sciences, Lorestan University, Khorram Abad, Iran.

aliasgharfarokhi66@yahoo.com

A. Nazari ${ }^{\otimes}$

Faculty of Mathematical Sciences, Lorestan University, Khorram Abad, Iran.

nazari.ar@lu.ac.ir

Received: April 22, 2018

Accepted: November 14, 2018 